\title{
Scale-invariance of black hole accretion: modeling emission from a black hole X-ray binary with relativistic accretion flow simulations
}

\author{
M. Mościbrodzka ${ }^{1 \star}$ \\ Department of Astrophysics/IMAPP, Radboud University, P.O. Box 9010, 6500 GL Nijmegen, The Netherlands
}

Accepted XXX. Received YYY; in original form ZZZ

\begin{abstract}
We model non-thermal emission spectrum of the extremely sub-Eddington X-ray binary system A0620-00. It is believed that this non-thermal emission is produced by a radiatively inefficient "quiescent" accretion onto a stellar-mass black hole present in the system. We post-process GRMHD simulations with multiwavelength, fully polarized, relativistic radiative transfer calculations to predict broadband spectra and emission polarization levels for a range of electron models and accretion rates. We find that a model with strong coupling of electrons and ions in the accretion disk and accretion rate of only $\dot{M}=3 \times 10^{-13}\left[\mathrm{M}_{\odot} \mathrm{yr}^{-1}\right]$ is able to recover the observed X-ray spectral slope as well as the excess of linear polarization detected in the source in NIR/optical bands. Our models constrain the spectral properties of a putative relativistic jet produced in this system. In addition we show that the magnetized winds from our hot accretion flow carry away a small fraction of the orbital angular momentum of the binary unable to explain the observed rapid orbital decay of the system. Similar to the present GRMHD simulations are often used to explain emission from sub-Eddington supermassvie black holes in Sgr A* or M87; the present simulations allow us to test whether some aspects of the quiescent black hole accretion are scale invariant.
\end{abstract}

Key words: black hole physics - MHD - polarization - radiative transfer - relativistic processes

\section{INTRODUCTION}

A0620-00 is a low mass X-ray binary system composed of a main sequence K-type star (V616 Mon) in orbit around a compact object. The orbital period of the system $P \approx 7.7$ $\mathrm{hr}$ derived from variations in optical light curves yields the separation between the two components $\sim 3-5 R_{\odot}$. The ellipsoidal shape of the optical light curve from the stellar companion indicates the tidal deformation of the star (McClintock \& Remillard 1986); the shape of optical light-curves are used to model the mass of the companion and the inclination of the system with respect to our line of sight. Cantrell et al. 2008 carried out a comprehensive analyses of 30 years of optical observations of this system and estimated the mass of the compact component to be $M_{\mathrm{BH}}=6.6 \pm 0.25 \mathrm{M}_{\odot}$ strongly favoring a black hole and the inclination of the system is $51 \pm 0.9 \mathrm{deg}$ (however, see e.g., Shahbaz et al. 1994, van Grunsven et al. 2017 for alternative evaluations). Notice that the precise determination of $M_{\mathrm{BH}}$ and $i$ depends on the uncertain details of the optical emission produced by

* E-mail: m.moscibrodzka@astro.ru.nl a putative accretion disk around the accretor and emission from a hot spot at the outer edge of the disk (Neilsen et al. 2008). Above $M_{\mathrm{BH}}$ and $i$ yield distance to the system to be $D=1.06 \pm 0.12 \mathrm{kpc}$, making A0620-00 the closest currently known (stellar-mass) black hole system to Earth (Cantrell et al. 2010).

These detailed studies of A0620-00 followed X-ray nova outburst in the source in 1975 when the source become the brightest X-ray source on the sky for a few days $\left(L_{\mathrm{X}} \approx 10^{38}[\mathrm{ergs} / \mathrm{s}]\right.$, Elvis et al. 1975). Since the outburst the source X-ray luminosity of A0620-00 is extremely low $L_{\mathrm{X}} \leq 10^{31}$ (Kong et al. 2002) and the system is detectable in X-rays due to its proximity. In units of Eddington luminosities (where Eddington luminosity is defined as usually: $L_{\mathrm{Edd}} \equiv 4 \pi G M_{B H} m_{p} c / \sigma_{\mathrm{TH}}=1.26 \times$ $\left.10^{38}\left(M_{\mathrm{BH}} / M_{\odot}\right)\left[\mathrm{ergs} \mathrm{s}^{-1}\right]\right)$, A0620-00 is a strongly subEddington source, $L_{\mathrm{X}} \leq 10^{-7} L_{\mathrm{Edd}}$ (Dinçer et al. 2018) that is usually classified as a quiescent phase of accretion. In this phase the accretion disk around the black hole is believed to take form of a radiatively inefficient/advection dominated flow (RIAF/ADAF, Yuan \& Narayan 2014). There are only a few other known black hole systems in strongly 
sub-Eddington state: XTE J1118+480 $\left(L_{\mathrm{X}} / L_{\mathrm{Edd}}=4 \times 10^{-9}\right.$, Gallo et al. 2014), Swift J1357.2-0933 ( $L_{\mathrm{X}} / L_{\mathrm{Edd}}=4 \times 10^{-9}-$ $3 \times 10^{-8}$, Armas Padilla et al. 2014; Plotkin et al. 2016), and Sgr A* associated with supermassvie black hole in the center of our Galaxy $\left(L_{\mathrm{X}} / L_{\mathrm{Edd}}<10^{-10}\right.$, Baganoff et al. 2003). Besides optical/NIR and X-ray monitoring, A0620-00 system is frequently observed in radio (Gallo et al. 2006) and recently Atacama Large Millimeter Array has detected the millimeter counterpart of the system (Gallo et al. 2019).

The most prominent component of A0620-00 multiwavelenght spectrum is a optical/NIR thermal hump produced predominantly by the companion star with some contribution of non-thermal emission from accretion processes. The non-stellar emission in radio/mm is believed to originate in a relativistic outflow/jet. The X-ray emission is thought to be produced by synchrotron process (via direct synchrotron or self-synchrotron Compton emission) at the jet base and/or by a RIAF/ADAF launching the jet. The details of the jet structure and the jet launching region, the strength of magnetic fields and particle acceleration, are constrained only in terms of simplified semi-analytic models (e.g., Plotkin et al. 2015; Connors et al. 2017).

In this work, we use a global model of relativistic hot magnetized accretion disk to model non-thermal emission from A0620-00 system. The multiwavelenght emission spectra are calculated using selfconsistent models of accretion realized via three dimensional general relativistic magnetohydrodynamics (3D GRMHD) numerical simulations and self-consisitent general relativistic radiative transfer models. The main motivation for discussing the accretion onto black holes in X-ray binary system in context of a more detailed (compared to all previous ones) models are the recent results from the Event Horizon Telescope (EHT). The telescope mapped a hot accretion flow around sub-Eddington supermassive black hole in M87 galaxy on horizon scales (Event Horizon Telescope Collaboration et al. 2019a) and GRMHD models of radiatively inefficient accretion have been used to interpret the observations (Event Horizon Telescope Collaboration et al. 2019b). The major uncertainty in these GRMHD models was the thermodynamics of electrons that allowed various different physical scenarios to be acceptable. The same uncertainty concerns future modeling of Sgr A*, which is the second target for EHT. It is believed that black holes of stellar and supermassive masses accrete and produce jets in the same way at a given accretion rate scaled to their Eddington limit (Merloni et al. 2003, Falcke et al. 2004). Accretion flow around the stellar-mass black hole in A0620-00 is not only another ideal source for observational tests of electron thermodynamics in GRMHD models that may help us to understand the general structure of strongly sub-Eddington flows but also, together with EHT results, allows us to test and understand the similarities of physical processes nearby supermassvie and stellar-mass black hole horizons.

The structure of the paper is as follows. In Sect. 2 we describe details of our numerical modeling techniques and adopted physical scenario of accretion. In Sects. 3 and 4, we present the results of the modeling. We discuss the results in Sect. 5.

\section{MODEL OF POLARIZED MULTIWAVELENGHT EMISSION FROM ACCRETING BLACK HOLE}

In quiescent state, the dynamics of RIAF/ADAF and putative jet is unaffected by radiative losses (Ryan et al. 2017). Hence A0620-00 can be considered in terms of a nonradiative GRMHD flow models.

We integrate the equations of GRMHD starting with the following initial and boundary conditions. Our initial distribution of plasma rest-mass density and internal energy is described by an analytical model of a torus in Keplerian orbit around a black hole (Fishbone \& Moncrief 1976). Two parameters describe the initial torus size: the radius of pressure maximum $r_{\max }=15.05 G M / c^{2}$ and its inner edge $r_{i n}=6.4 G M / c^{2}$ where $G M / c^{2}$ is the gravitational radius of the black hole of mass $M$. The torus is seeded with poloidial magnetic fields which geometry is described by vector potential $A_{\phi} \sim \rho^{2} r^{4}$, where $\rho$ is the rest-mass density of plasma and $r$ is a radial distance from the center of the coordinate system. $A_{\phi}$ sets the initial geometry of the magnetic fields and magnetic field strength is specified via $\beta$ plasma parameter defined as the ratio of gas to magnetic pressure, $\beta=P_{\text {gas }} / P_{\text {mag. }}$. In the current model at time $t=0$ the field is weak with maximum value of $\beta_{\max }(t=0)=100$.

The spin of the black hole ${ }^{1}$ is a key parameter of the model that governs the shape of the emission spectrum (e.g., Mościbrodzka et al. 2009). There exist constraints on spin of A0620-00 black hole $\left(a_{*}=0.12 \pm 0.19\right)$ based on thin-disk continuum fitting method when the source was in outburst in 1975 (Gou et al. 2010). Although these constraints are model dependent, they motivate our fiducial simulation that assumes Schwarzschild black hole.

Using public version of GRMHD code harm (harmpi), we integrate the equations of general relativistic magnetohydrodynamics in Schwarzschild metric on a threedimensional, spherical-polar grid that extends from within the event horizon of the black hole to $50 \mathrm{GM} / \mathrm{c}^{2}$. The computational grid is spaced equally in $\log (\mathrm{r})$, in polar $(\theta)$ and azimuthal $(\phi)$ angle direction with moderate resolution $\left(\mathrm{N}_{\mathrm{r}} \mathrm{xN}_{\theta} \mathrm{xN}_{\phi}\right)=(192 \times 192 \times 192)$. The inner boundary of the simulation is separated from the computational domain by the event horizon of the black hole. At the outer radius we apply outflow conditions. We follow the evolution of the torus until $t_{f}=3200 G M / c^{3}$ i.e. until the accretion flow settles down to a steady-state phase. In the steady-state phase, the accretion flow near the equatorial plane is turbulent, where the turbulence develops in the initial torus via the magnetorotational instability (Balbus \& Hawley 1998, Gammie et al. 2003). In the polar regions, we observe coherent poloidal magnetic fields that diffused there with plasma from the initial torus. To put the current model in context of earlier works, we calculate the magnitude of the magnetic field flux that accumulates near the event horizon, $\phi_{B}=\int B^{r} d A / \sqrt{\dot{M} r_{g}^{2} c}$ (the quantity is most useful when defined in dimensionless units). Models with $\phi_{B} \sim 1$ are usually refered as to standard and normal evolution (SANE)

1 The dimensionless spin parameter is defined as usual: $a_{*} \equiv$ $J c / G M^{2}$ with $\left|a_{*}<1\right|$ where $M$ and $J$ are, respectively, the mass and the angular momentum of a black hole. 

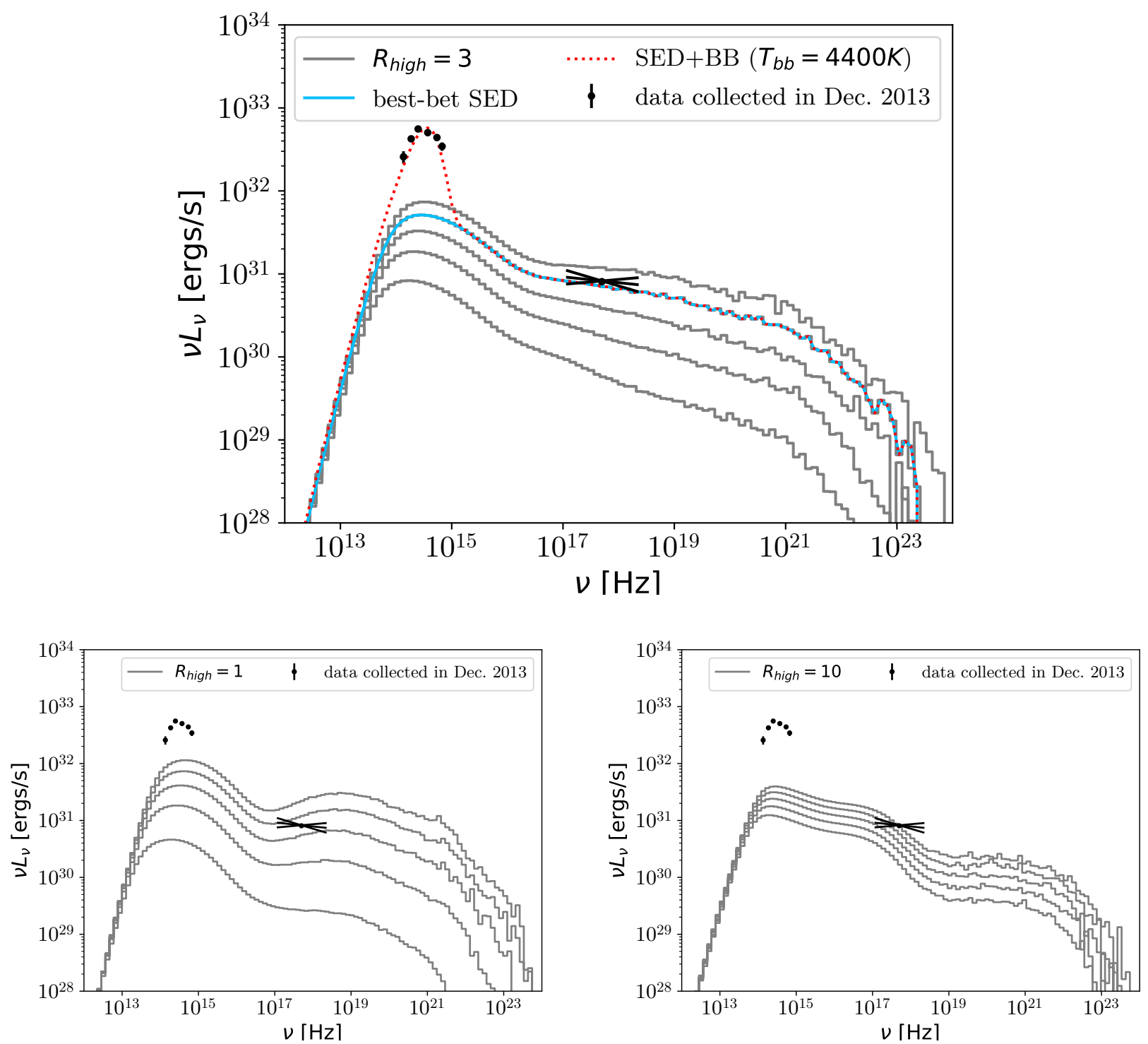

Figure 1. Spectral energy distribution of synchrotron and self-synchrotron Compton emission produced in GRMHD simulations of hot accretion flow onto a stellar mass black hole in X-ray binary system. All models assume that the viewing angle of the system is $\sim 50$

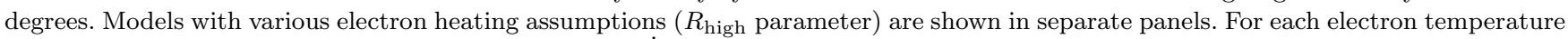
prescription we show spectra assuming a few values of $\dot{M}$ to find model that best matches X-ray flux and spectral slope. Model spectra are overplotted with observational data of A0620-00 system collected in 2013 (Dinçer et al. 2018). Model that recovers the X-ray emission characteristics is marked with a solid blue line. Best-bet model with thermal stellar spectrum added is shown as red dashed line.

models (e.g., Porth et al. 2019) and those with $\phi_{B} \sim 15$ as to magnetically arrested disks (MADs) (e.g., Tchekhovskoy et al. 2011). In the SANE scenario, the accretion disk is turbulent which results in small variations in accretion rate. In the MAD scenario, one expects the accretion flow to be occasionally halted by strong magnetic forces. MADs also produce jets that are more powerful compared to those produced by SANEs. We find that in our model $\phi_{B} \lesssim 8$ meaning that our model is somewhere in-between the two distinct regimes of accretion. The quantity $\phi_{B}$ can be changed by varying the size of the initial torus or by assigning different initial field geometry/strength, but it cannot be easily pre- dicted. The current setup of initial magnetic fields is chosen arbitrarily as there is no model-independent observational constraints on the magnetic fields in A0620-00 system.

We construct mock multiwavelenght spectral energy distributions (SEDs) based on the accretion model using postprocessing fully polarized general relativistic radiative transfer schemes (Noble et al. 2007; Dolence et al. 2009; Mościbrodzka \& Gammie 2018, and Moscibrodzka in prep.). The radiative processes included are: synchrotron emission and self-synchrotron Compton (SSC).

The primary parameter of our radiative transfer model is the distribution function of radiating electrons. At low 
accretion rates the timescale for thermal coupling between electrons and ions is much longer compared to the dynamical timescale of the flow leading to two-temperature plasma (Mahadevan \& Quataert 1997). Moreover, electron acceleration may produce a power-law distribution function. Predicting radiative characteristics of our simulation from first principles would require a sub-grid prescription for the evolution of electron distribution function (Ressler et al. 2015; Ryan et al. 2017; Rowan et al. 2017; Chael et al. 2018). In the present work we adopt a simpler approach. We assume that electrons in our simulations have relativistic, thermal (Maxwell-Jüttner) distribution function and that the plasma has a two-temperature structure in which electron and ions temperature coupling depends on local plasma magnetization $\left(\beta \equiv P_{\text {gas }} / P_{\text {mag }}\right)$. This approach is motivated by particle-in-cell simulations of collisionless plasma (Kawazura et al. 2018). Following Mościbrodzka et al. 2016, we calculate $T_{\mathrm{e}}$ from formulae that describes coupling of ion-to-electron temperatures: $T_{\mathrm{i}} / T_{\mathrm{e}} \equiv R_{\mathrm{high}} \beta^{2} /\left(1+\beta^{2}\right)+1 /\left(1+\beta^{2}\right)$ where $T_{\mathrm{i}}$ is the ion temperature followed in the GRMHD model and where $R_{\text {high }}$ is the free parameter of the model that is constrained by fitting the model SED to the observed spectrum. Notice that by increasing $R_{\text {high }}$ the decoupling of electron and ions in the weakly magnetized plasma is stronger and electrons may become subrelativistic.

\section{SCALING GRMHD SIMULATION TO A0620-00}

The procedure of scaling GRMHD simulation to black hole in X-ray binary is similar to the modeling emission from supermassive black holes: Sgr A* or M87 (Mościbrodzka et al. 2009, 2016). Given black hole mass $M_{\mathrm{BH}}=6.6 \mathrm{M}_{\odot}$, viewing angle $i=51 \mathrm{deg}$ and the electron model, we fit model SED to the observational data by adjusting the accretion rate onto the black hole, $\dot{M}$. However, instead of fitting emission at mm-waves (as it is usually done in the case of these Sgr $A^{*}$ and M87 because the sources are best resolved in mm-waves) we find the model free parameters by fitting the model spectrum to X-ray flux and X-ray spectral slope. It should be understood that here we assume that the X-ray emission is produced by SSC on thermal relativistic electrons in the accretion inflow/outflow, which in generally does not have to be the case (see e.g., Connors et al. 2017 for alternative scenario where X-rays are produced directly by synchrotron emission from accelerated particles). In this work, we also do not model radio/ $\mathrm{mm}$ emission from the large scale jet because our simulations are designed for modeling near horizon emission. The observed emission in $\mathrm{GHz}$ and lower frequencies, due to self-absorption effects, is expected to be produced at the distances $r>10^{3} G M / c^{2}$ from the black hole (Blandford \& Königl 1979).

Although GRMHD simulations are intrinsically variable, here we do not model variability of the source. The duration of entire GRMHD simulation is $3200 G M / c^{3} \approx 0.1$ second, significantly shorter compared to $7.7 \mathrm{~h}$ orbital period. Hence, we pick a few snapshots at the end of the simulation time and assume that they are representing average state of the accretion flow in the system.

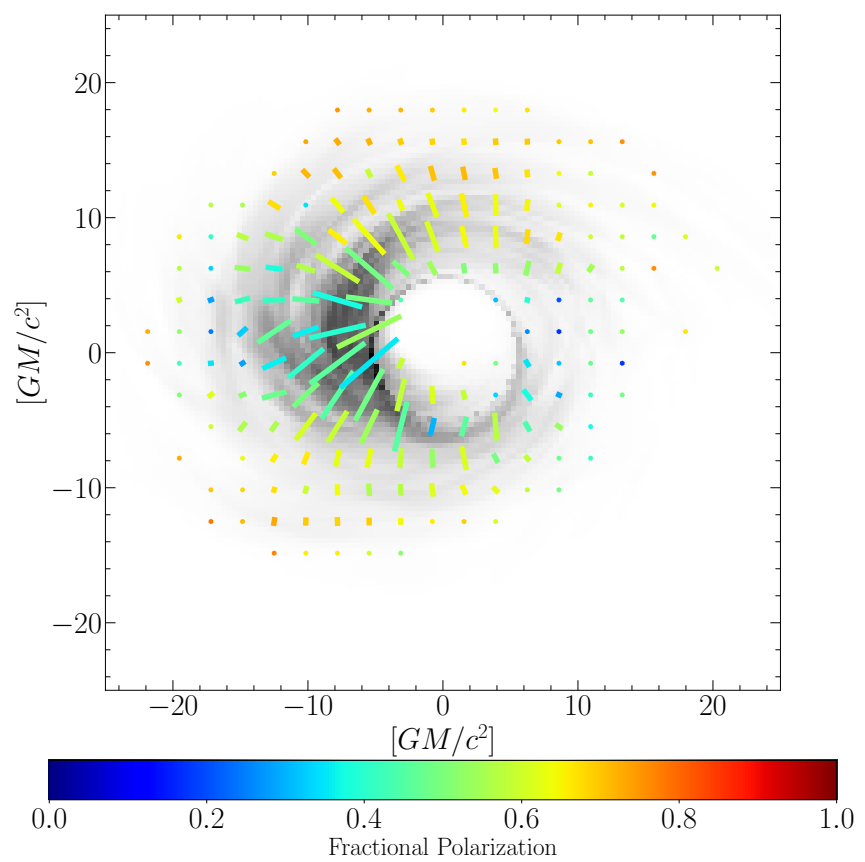

Figure 2. NIR ( $K_{s}$ band) resolved image of the GRMHD simulation scaled to A0620-00 system. Total intensity is shown in gray scale (we mask regions with $<1$ per cent of maximum intensity). The color ticks show polarization of the model: the tick length is proportional to polarized flux, the tick color is fractional linear polarization and tick orientation is EVPA. We cannot resolve accretion flows onto stellar mass black hole on this scales so this image is shown only to help understand the total polarization and EVPA in the system. The synchrotron emission is intrinsically highly linearly polarized and shows organized EVPAs but the total linear polarization is beam-depolarized to less than 10 per cent.

\section{RESULTS}

In Fig. 1 we show our model SEDs overploted with the black hole X-ray binary observational data collected in 2013, published in Dinçer et al. (2018). The X-ray data in Fig. 1 is Chandra observation no. 14656. Notice that prior to 2013 the X-ray luminosity of the system was weaker by a factor of 2 (in 2005) and 6 (in 2000) but the spectral slope has not changed significantly from 2000 to 2013 . This issue is further discussed in Sect. 5.

In Fig. 1, we show SEDs for three values of $R_{\text {high }}=1,3$ and 10 and a few values of accretion rates $\dot{m}$ for each $R_{\text {high }}$ $\left(\dot{m}=4 \times 10^{-7}-2 \times 10^{-6}\right.$ for $R_{\text {high }}=1 ; \dot{m}=8 \times 10^{-7}-2.5 \times$ $10^{-6}$ for $R_{\text {high }}=3$; and $\dot{m}=2.5 \times 10^{-6}-4 \times 10^{-6}$ for $R_{\text {high }}=$ 10 , where $\dot{m}=\dot{M} / \dot{M}_{\mathrm{Edd}}$ and $\left.\dot{M}_{\mathrm{Edd}}=1.38 \times 10^{-7}\left[\mathrm{M}_{\odot} \mathrm{yr}^{-1}\right]\right)$.

In Fig 1, we show that for a given electron model the changes of accretion rate shifts the spectrum in vertical direction. The X-ray spectral slope however is sensitive to the adopted electron heating scenario. As expected increasing $R_{\text {high }}$ (cooler electrons in the disk plane) results in softer Xray spectra. Within our grid of models, the model that best describes the X-ray luminosity and the X-ray spectral slope is the one with $R_{\text {high }}=3$ and $\dot{m}=2 \times 10^{-6}$. The best-bet model is marked in Fig. 1 with a blue solid line. All models 
with $R_{\text {high }}=1$ the spectral slope in X-ray is positive and for $R_{\text {high }}=10$ it becomes too steep compared to observations.

The observed NIR/optical emission is dominated by light from the stellar companion which is not included in the simulation. We model this spectral component with the Planck function, parameterized by a black-body temperature $T_{\mathrm{bb}}$, integrated over the surface of the star with radius $R_{*}=0.75 R_{\odot}$. This simple approach does not include effects of deformation of the star by tidal forces or orbital phase of the star. In the best-bet model the non-thermal emission from hot accretion flow produces $\sim 10$ per cent of the optical/NIR flux, the thermal emission from the stellar atmosphere requires $T_{\mathrm{bb}}=4400 \mathrm{~K}$.

The radiation models are fully polarized so we can report the polarization of our best-bet model in NIR/optical band and compare it to A0620-00 polarimetric data found in the literature (Russell et al. 2016). Accretion rate controls optical thickness of the model, $\tau_{\nu}$. In our best-bet model, $\tau_{\text {NIR/optical }} \sim 1$ and it is produced near the event horizon of the black hole. The polarization of light from an optically thin regions does not have to be strongly polarized as the plasma can still be Faraday thick due to some cold electrons present in the disk (Mościbrodzka et al. 2017) or it can be simply beam depolarized. In our best model, the total fractional linear polarization $\left(m \equiv \sqrt{Q_{\text {tot }}^{2}+U_{\text {tot }}^{2}} / I_{\text {tot }}\right.$, where $I, Q$, and $U$ are the Stokes parameters and subscript 'tot' mean image integrated, see next section and Fig. 2) modeled in $K_{s}, \mathrm{H}, \mathrm{J}$ and $\mathrm{Z}$ bands are $m=9.3,9.11,8.9,8.9$ per cent and in $\mathrm{V}, \mathrm{B}$, and $\mathrm{U}$ filters $m=9,9.5$, and 10 per cent.

If we assume that the stellar emission is unpolarized and that the non-thermal emission is a fraction $f$ of the total opt/NIR flux, then the total observed fractional polarization is $m_{\text {obs }} \equiv m f /(1+f)$. Our model predicts $f=0.1$ which yields $m_{\mathrm{obs}} \approx 1$ per cent in both NIR and optical bands. This predicted fractional polarization is consistent with the observed (intrinsic, i.e., with subtracted polarization by interstellar dust) $m \approx 1$ per cent in the NIR/optical bands (Russell et al. 2016). It is therefore possible that the observed level of polarization is produced by a hot accretion flow onto the black hole but this low polarization does not have to indicate tangled/chaotic magnetic fields as suggested by Russell et al. (2016). As evident in Fig. 2, at the assumed viewing angle of $51 \mathrm{deg}$ the resolved EVPAs (where $E V P A \equiv 0.5 \arg (Q+i U)$ are approximately radial and track the toroidal component of magnetic fields in the accretion disk. The total (i.e., image integrated) position angles $\left(E V P A_{t o t} \equiv 0.5 \arg \left(Q_{t o t}+i U_{t o t}\right)\right.$ and we use convention in which $\mathrm{EVPA}=90 \mathrm{deg}$ would be in horizontal position) change from $174 \mathrm{deg}$ in NIR to $133 \mathrm{deg}$ in optical. Interestingly, our best-bet model also predicts that total fractional circular polarization should vary from -1.65 percent in NIR to -0.25 percent in optical window. In models, the circular polarization is produced by the Faraday conversion by relativistic electrons.

The mass accretion rate estimated from our model fit-

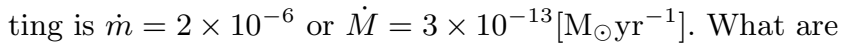
the typical densities and magnetic field strength in models scaled to this accretion rate? In Fig. 3, we show maps of particle number density, magnetic fields strength, and electron temperatures in the GRMHD used to produce the best-bet SED. Near horizon the $n_{\mathrm{e}} \approx 10^{14}\left[\right.$ particles $/ \mathrm{cm}^{3}$ ] and $B \approx 10^{6}[\mathrm{G}]$. These numbers are consistent with those estimated by Connors et al. (2017) based on modeling observational data from 2005.

\section{DISCUSSION}

In this paper, we model the non-thermal (synchrotron and $\mathrm{SSC}$ ) emission from accreting black hole in X-ray binary system using GRMHD models of accretion flows. This work focuses on the extremely sub-Eddington system A0620-00 one of the few systems where the weakly radiating GRMHD models are applicable (for similar emission models but in more luminous sources in "hard/low" state see O' Riordan et al. 2016). In our analyses, we model X-ray luminosity, Xray spectral slope and, for the first time, also NIR/optical polarization of the non-thermal emission to constrain the electron heating model and accretion rate onto the black hole.

We find that to recover X-ray flux and the X-ray spectral slope rather strong coupling between ions and electrons in the accretion disk $\left(R_{\text {high }}=3\right)$ is favored. The corresponding mass accretion rate is $\dot{M}=3 \times 10^{-13}\left[\mathrm{M}_{\odot} \mathrm{yr}^{-1}\right]$. For these parameters, our radiative model predicts levels of observed fractional linear polarization that are consisitent with those observed in NIR/optical bands. Since the model X-ray spectral slope weakly depends on $\dot{M}$ and strongly depends on $R_{\text {high }}$, we expect that fitting model SEDs to Xray data from 2000/2005 would not change our conclusion regarding the favored value of $R_{\text {high }}$. However, we expect that model with mass accretion rate 2-3 times lower than $\dot{M}=3 \times 10^{-13}\left[\mathrm{M}_{\odot} \mathrm{yr}^{-1}\right]$ would be required to match six and two times weaker X-ray emission detected in 2000 and 2005, respectively.

In the current model, the synchrotron emission peaks in NIR/optical band with flux $F_{\text {peak }}=260[\mu \mathrm{Jy}]$. At $\nu<\nu_{\text {peak }}$, the model spectrum steeply declines only due to small size of our simulation. Although our simulation does not include a large scale jet - the radio observations of A0620-00 still constrain some properties of an eventual jet model. Given that the observed radio emission is at the level of $F_{10 G H z} \approx$ $15-50[\mu \mathrm{Jy}]$ (Gallo et al. 2019), any extended jet originating from our magnetized disk model should produce a spectrum $F_{\nu} \sim \nu^{-p}$ with slightly inverted slope $p=0.3-0.17$ to match the observations.

An independent approach to constrain the strength of magnetic fields in the accretion flow models in binary systems is by measuring how much angular momentum is lost from the system via magnetized outflows and estimating the corresponding orbital decay in the system due to magnetic braking. The orbital decay in A0620-00 is rapid, with orbitalperiod derivative $\dot{P}=-0.6\left[\mathrm{~ms} \mathrm{yr}^{-1}\right]$ (González Hernández et al. 2014) and it cannot be explained by the emission of gravitational waves alone. Magnetic braking of the system is a possible explanation for the measured $\dot{P}$ but other explanations, such as resonant interactions between the binary and the possible circumbinary disk, has been proposed (Chen \& Podsiadlowski 2017). Here we can estimate the magnitude of the magnetic braking of the system using first-principles approach. The orbital angular momentum of a binary system is $J_{\text {orb }}=M_{\mathrm{BH}} M_{*} /\left(M_{\mathrm{BH}}+M_{*}\right) \sqrt{G\left(M_{\mathrm{BH}}+M_{*}\right) d}$ where $d$ is a separation between the star and the black hole. In GRMHD 

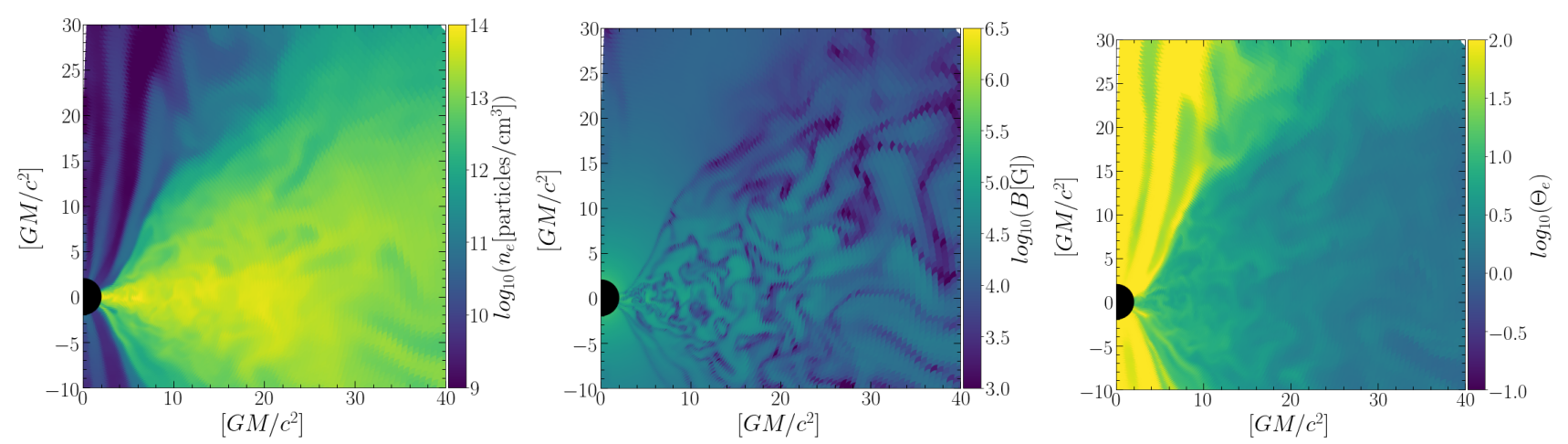

Figure 3. Maps of particle number density, magnetic field strengths and electron temperatures (in units of electron rest mass) in the model scaled to A0620-00 system. The black circle marks the event horizon of a Schwarzschild black hole. The plasma density and strength of magnetic fields near the event horizon are roughly consistent with those estimated by Connors et al. (2017) based on various semi-analytic jet models with simplified geometries.

simulations the loss of total angular momentum through the outer boundary is defined as: $\dot{J}\left(r_{\text {out }}, t\right)=\int_{\theta} \int_{\phi} T_{\phi}^{r} d A_{\theta \phi}$, where $T_{\phi}^{r} \equiv\left(\rho+\gamma u+b^{2}\right) u^{r} u_{\phi}-b^{r} b_{\phi}$ is the stress-energy tensor describing the radial flux of angular momentum. The quantity $u$ is the internal energy of the gas, $\gamma=4 / 3$ is the adiabatic index, $u^{\mu}$ is the four-velocity of the gas and $b^{\mu}$ is a four-vector which describes magnetic field in a frame comoving with the gas. We integrate the above formula at $r_{\text {out }}=50 G M / c^{2}$ over $\theta \in(0, \pi)$ and $\phi \in(0,2 \pi)$. In our best-bet model the ratio of angular momentum flux through the outer boundary to the orbital angular momentum is extremely low $\dot{J} / J_{\text {orb }} \approx 10^{-22}[1 / \mathrm{s}]$, which could account for orbital period derivative of $\dot{P}=2 \times 10^{-8}\left[\mathrm{~ms} \mathrm{yr}^{-1}\right]$ only. Our calculations confirm that the inner highly magnetized, rotating accretion flow (with current $\dot{M}$ ) alone cannot be responsible for the rapid orbital decay observed in the system and favor the idea of a circumbinary disk.

How does the current picture of a quiescent accretion flow onto a stellar-mass black hole compares to the ones around supermassive black holes in e.g., Sgr A*? Sgr A* synchrotron emission at millimeter wavelengths is relatively highly polarized (Bower et al. 2018) suggesting that in the quiescent state the electrons strongly couple to ions in RIAFs/ADAFs (see also Bower et al. 2019 for additional constraints on electron temperatures based on the shape of Sgr A* Terahertz spectrum) regardless of the black hole mass. If the relativistic flow around the black hole was made of two-temperature plasma containing sub-relativistic electrons $\left(R_{\text {high }} \gg 1\right)$ we would observe the depolarization the synchrotron emission via strong Faraday effects intrinsic to the accretion flow itself (Mościbrodzka et al. 2017; JiménezRosales \& Dexter 2018). Fitting models with $R_{\text {high }} \gg 1$ in both sources would require considering a model for electron acceleration into a power-law function to recover both: the X-ray spectral slope in A0620-00 and the significant polarization of synchrotron emission in Sgr A*.

Finally, we have considered a single specific GRMHD simulation. A wide survey of GRMHD simulation parameter space (including different values of black hole spin) should be carried out for both A0620-00 and Sgr A* to carefully test the scale-invariance paradigm. Ultimately, the future EHT polarimetric images of Sgr A* (and M87) on event hori- zon scales would greatly help us to constraint the magnetic field geometries, electron distribution functions and accretion rates in these sources. In A0620-00 the excess polarization could be also produced in a circumbinary disk illuminated by both companion star and accretion flow (Muno \& Mauerhan 2006). The latter additionally constrains the electron heating models in RIAFs/ADAFs and should be taken into account in the future works.

\section{ACKNOWLEDGEMENTS}

This research has made use of NASA's Astrophysics Data System Bibliographic Services. I thank Sasha Tchekhovskoy for his comments.

\section{REFERENCES}

Armas Padilla M., Wijnands R., Degenaar N., Muñoz-Darias T., Casares J., Fender R. P., 2014, MNRAS, 444, 902

Baganoff F. K., et al., 2003, ApJ, 591, 891

Balbus S. A., Hawley J. F., 1998, Reviews of Modern Physics, 70, 1

Blandford R. D., Königl A., 1979, ApJ, 232, 34

Bower G. C., et al., 2018, ApJ, 868, 101

Bower G. C., et al., 2019, ApJ, 881, L2

Cantrell A. G., Bailyn C. D., McClintock J. E., Orosz J. A., 2008, ApJ, 673, L159

Cantrell A. G., et al., 2010, ApJ, 710, 1127

Chael A., Rowan M., Narayan R., Johnson M., Sironi L., 2018, MNRAS, 478, 5209

Chen W.-C., Podsiadlowski P., 2017, ApJ, 837, L19

Connors R. M. T., et al., 2017, MNRAS, 466, 4121

Dinçer T., Bailyn C. D., Miller-Jones J. C. A., Buxton M., MacDonald R. K. D., 2018, ApJ, 852, 4

Dolence J. C., Gammie C. F., Mościbrodzka M., Leung P. K., 2009, ApJS, 184, 387

Elvis M., Page C. G., Pounds K. A., Ricketts M. J., Turner M. J. L., 1975, Nature, 257, 656

Event Horizon Telescope Collaboration et al., 2019a, ApJ, 875, L4

Event Horizon Telescope Collaboration et al., 2019b, ApJ, 875, L5

Falcke H., Körding E., Markoff S., 2004, A\&A, 414, 895

Fishbone L. G., Moncrief V., 1976, ApJ, 207, 962 
Gallo E., Fender R. P., Miller-Jones J. C. A., Merloni A., Jonker P. G., Heinz S., Maccarone T. J., van der Klis M., 2006, MNRAS, 370, 1351

Gallo E., et al., 2014, MNRAS, 445, 290

Gallo E., et al., 2019, MNRAS, p. 1627

Gammie C. F., McKinney J. C., Tóth G., 2003, ApJ, 589, 444

González Hernández J. I., Rebolo R., Casares J., 2014, MNRAS, 438, L21

Gou L., McClintock J. E., Steiner J. F., Narayan R., Cantrell A. G., Bailyn C. D., Orosz J. A., 2010, ApJ, 718, L122

Jiménez-Rosales A., Dexter J., 2018, MNRAS, 478, 1875

Kawazura Y., Barnes M., Schekochihin A. A., 2018, arXiv eprints,

Kong A. K. H., McClintock J. E., Garcia M. R., Murray S. S., Barret D., 2002, ApJ, 570, 277

Mahadevan R., Quataert E., 1997, ApJ, 490, 605

McClintock J. E., Remillard R. A., 1986, ApJ, 308, 110

Merloni A., Heinz S., di Matteo T., 2003, MNRAS, 345, 1057

Mościbrodzka M., Gammie C. F., 2018, MNRAS, 475, 43

Mościbrodzka M., Gammie C. F., Dolence J. C., Shiokawa H., Leung P. K., 2009, ApJ, 706, 497

Mościbrodzka M., Falcke H., Shiokawa H., 2016, A\&A, 586, A38

Mościbrodzka M., Dexter J., Davelaar J., Falcke H., 2017, MNRAS, 468, 2214

Muno M. P., Mauerhan J., 2006, ApJ, 648, L135

Neilsen J., Steeghs D., Vrtilek S. D., 2008, MNRAS, 384, 849

Noble S. C., Leung P. K., Gammie C. F., Book L. G., 2007, Classical and Quantum Gravity, 24, S259

O' Riordan M., Pe'er A., McKinney J. C., 2016, ApJ, 819, 95

Plotkin R. M., Gallo E., Markoff S., Homan J., Jonker P. G., Miller-Jones J. C. A., Russell D. M., Drappeau S., 2015, MNRAS, 446, 4098

Plotkin R. M., et al., 2016, MNRAS, 456, 2707

Porth O., et al., 2019, arXiv e-prints, p. arXiv:1904.04923

Ressler S. M., Tchekhovskoy A., Quataert E., Chandra M., Gammie C. F., 2015, MNRAS, 454, 1848

Rowan M. E., Sironi L., Narayan R., 2017, ApJ, 850, 29

Russell D. M., Shahbaz T., Lewis F., Gallo E., 2016, MNRAS, 463, 2680

Ryan B. R., Ressler S. M., Dolence J. C., Tchekhovskoy A., Gammie C., Quataert E., 2017, ApJ, 844, L24

Shahbaz T., Naylor T., Charles P. A., 1994, MNRAS, 268, 756

Tchekhovskoy A., Narayan R., McKinney J. C., 2011, MNRAS, 418, L79

Yuan F., Narayan R., 2014, ARA\&A, 52, 529

van Grunsven T. F. J., Jonker P. G., Verbunt F. W. M., Robinson E. L., 2017, MNRAS, 472, 1907

This paper has been typeset from a $\mathrm{T}_{\mathrm{E}} \mathrm{X} / \mathrm{L}_{\mathrm{A}} \mathrm{T}_{\mathrm{E} X}$ file prepared by the author. 